\title{
System Simulation of Driving Mechanism of Rural Tourism Development Based on Data Mining Analysis and Cloud Computing
}

\author{
Mingtong Li $\mathbb{B D}^{1,2}$ \\ ${ }^{1}$ College of Management, China University of Mining and Technology, Xuzhou 221116, China \\ ${ }^{2}$ School of Architecture, Yantai University, Yantai 264005, China \\ Correspondence should be addressed to Mingtong Li; limingtong@ytu.edu.cn
}

Received 14 January 2022; Revised 8 February 2022; Accepted 9 February 2022; Published 4 March 2022

Academic Editor: Xin Ning

Copyright ( 2022 Mingtong Li. This is an open access article distributed under the Creative Commons Attribution License, which permits unrestricted use, distribution, and reproduction in any medium, provided the original work is properly cited.

\begin{abstract}
Rural tourism (RT) and cloud computing are important forces in implementing the rural revitalization strategy, and they play a key role in accelerating agriculture and rural area modernization, urban-rural integration, poverty alleviation in impoverished areas, and so on. RT's development is influenced by a variety of factors due to its high industrial relevance and strong driving function. The development of RT is examined from the perspectives of data mining and cloud computing in this paper, and a data mining model of the RT development's driving mechanism is built. Five driving modes of RT development are simulated by adjusting the parameters of industrial economy, resources, environment, and social culture in $\mathrm{H}$ city as an example. The simulation results are compared and analyzed in various modes. Finally, the coordinated development model of industrial economy, social culture, resources, and environment is found to be the best model of RT development in $\mathrm{H}$ city, and countermeasures and suggestions for RT development in H city are proposed based on the analysis results.
\end{abstract}

\section{Introduction}

The term RT (rural tourism) encompasses a wide range of concepts; the most important of which is the RT community, which is dependent on rural areas for tourist attractions, heritage and culture, rural activities, and rural life [1]. The interactive way and coordinated interactive procedure among various driving factors in the development of $\mathrm{RT}$ is the driving mechanism for its development. The dominant driving force of RT destinations is transformed as a result of differences in key influencing factors of RT development, changing the evolution path of destinations and the comparative advantages among destinations $[2,3]$. RT is closely linked to agricultural production, agricultural product processing, and rural service industries as an important branch of tourism. It is a new format that is aimed at improving rural social production, life, and ecology while also integrating the first, second, and third industries. It is an important way for China's rural revitalization strategy in the new era and is currently welcoming a period of strategic opportunities for development [4].

The research of driving mechanism is the key and difficult point of dialysis RT development. The research of driving factors and driving forces is the foundation of driving mechanism research, and the driving mechanism is its essence and core, which is conducive to comprehensively understanding and grasping the main driving factors, driving process and mechanism of RT development, coordinating the relationship between driving factors and driving forces, and thus promoting the healthy and sustainable development of tourist destinations $[5,6]$. As a complex whole system, the development of RT is influenced by many factors. The existence of an RT-driven mechanism is a structure, function, and interaction among various factors that promote the long-term and healthy development of the RT system structure and function [7]. The research on prosperity indicators of the eco-tourism industry can provide data reference for the government to formulate industrial transformation and 
upgrading policies by tracking and monitoring the trend of industrial operation and change, reflecting the trend of industrial development forward-looking, revealing and warning the dynamic data of industrial development risks [8]. Travelers have a high demand for the application of information and intelligence in the tourism industry, from accurate information search to personalized information recommendations. As a result, it is critical to employ data mining and machine learning technologies to mine tourism data resources and implement intelligent tourism $[9,10]$.

This paper closely focuses on the current frontier topics of China's new socialist countryside construction and rural socioeconomic development, and the topic selection is highly targeted, mainly involving how to solve the problems encountered in the development of RT in China, which has important theoretical and practical significance for promoting the healthy development of RT in China [11]. In this paper, taking RT in $\mathrm{H}$ city as an example, based on the driving mechanism model, the development of RT in $\mathrm{H}$ city is simulated and analyzed by using data mining method. Through scientific theoretical guidance and data analysis, the countermeasures and suggestions of RT development in $\mathrm{H}$ city are put forward. When formulating policies related to RT development, systematic thinking and methods should be adopted to analyze and make decisions to promote its sustainable and coordinated development, which has practical guiding significance.

\section{Related Work}

There is no accurate definition of tourism driving mechanism, which mainly refers to an action mechanism among driving forces in the process of tourism development, and it is an effective way to reveal the interaction of various parts of the tourism system. Literature [12] applied the theory of tourism-driven mechanism to domestic scholars in urban tourism research and established a dynamic model of urban tourism development which consists of four parts: tourism demand promotion, tourism gravity system, tourism development support system, and tourism intermediary system. Literature [13] based on the driving mechanism of RT, it puts forward four subsystems: demand power, cosupply power, support power, and media power and constructs the overall framework of RT driving mechanism. According to literature [14], tourist demand is the driving force of RT development, while RT products provided by rural destinations are the inducing force, emphasizing the push-pull effect of demand and supply. According to literature [15], the main driving forces for the development of RT are demand and supply, and RT is primarily located around the city, with outstanding rural and rural features. The beginning point of any behavior is "demand," and the end point is "meeting demand." The push-pull force formed by demand and supply, known as system power, can play a key role in the development of RT. According to demand and supply theory, literature [16] investigated the regional RT development situation and summarized the RT driving mechanism of the trinity of subjective driving mechanism, objective driving mechanism, and intermediate conditions.
According to literature [17], different development agents have different effects on the development driving mechanism, and each agent will form a corresponding operating mechanism, which will change depending on the stage of RT development. In general, the core of these studies has not left the two main driving forces of demand and supply, regardless of how the specific driving mechanism is constructed. The main driving forces in this system are interconnected, influence each other, and act in concert on the RT driving mechanism's operation process.

As a hot spot of tourism research at home and abroad, researchers have carried out various researches and discussions on RT. The research focus of RT abroad mainly focuses on RT supply, RT providers and their market segmentation, etc. As far as the driving mechanism of RT development is concerned, literature [18] holds that economic benefits, government funds, and policy support are the driving forces of RT. Literature [19] holds that the driving mechanism of RT development includes the attraction factor based on RT supply, the demand factor based on RT generating power, and the external guarantee based on RT development. Literature [20] holds that RT development mechanism includes government support mechanism, industrial chain mechanism, and farmer participation mechanism. The RT-driven mechanism constructed by reference [21] is composed of five subsystems: demand, supply, media, support, and decision-making. Using the BP neural network model and the grey system GM $(1,1)$ model, the future development trend of RT in a city is predicted. The tourism sustainable development system, according to literature [22], can be divided into four subsystems: population, resources, environment, and tourism. According to the literature [23], the development of tourism will cause local community residents' attitudes toward developing RT to diverge, affecting the residents' overall attitude toward tourism. Locals have two main attitudes toward tourists: one is welcoming, and the other is hostile. The other type is diametrically opposed. Residents in the area are concerned about traffic jams, congestion, rising prices, and pollution caused by tourism. Users who use text or photos to query scenic spots are recommended the same type of scenic spots that are similar to the query, according to literature [24]. When users use text or photos to query scenic spots, they are recommended the same kind of scenic spots that are similar to the query. According to the literature [25], personalized scenic spot recommendations can be made using a Bayesian network based on user behavior and relationships. In a Bayesian learning framework, literature [26] combines user orientation, tourism history, photos with geographical marks, user preferences, and user attributes to provide a complete tourism recommendation, while demographic data is also taken into account in personalized scenic spot and route recommendations.

\section{Research Method}

3.1. Analysis of Driving Mechanism of RT Development. The development of RT cannot be separated from RT resources. $\mathrm{RT}$ resources are a unified and harmonious RT landscape 
formed by long-term mutual contact and interaction between human and environment and an organic whole composed of natural elements and unnatural elements. Starting from the basic points of tourism resources, RT resources are the objects of tourism activities that can attract tourists to a certain extent, have comprehensive economic, social, and ecological benefits after development and utilization, meet the needs of tourists, and bring economic benefits to residents, enterprises, and governments in RT places.

The natural landscape, socioeconomic conditions, cultural characteristics, and other tourism products in rural areas are comprehensive services that meet the needs of consumers for "food, housing, transportation, travel, shopping, and entertainment" [12]. RT from the perspective of industrial integration can be summarized as follows: in the rural space, the natural landscape, socioeconomic conditions, cultural characteristics, and other tourism products in rural areas are comprehensive services that meet the needs of consumers for "food, housing, transportation, travel, shopping, and entertainment" [13]. RT includes natural landscapes, rural scenery, agricultural production activities, agricultural culture, rural folk customs, farmers' living habits, and other tourist attractions. RT can take the form of a series of activities such as watching, tasting, experiencing, exercising, staying fit, learning, conducting scientific research, shopping, and taking a vacation.

From the development process of RT, it can be seen that the main body of RT products is farmers. This is different from the actual situation of RT development in China. There are various types of subjects providing RT products in China, including not only farmers but also foreign tourism enterprises and individuals, with diversified characteristics. Through the above analysis, this paper holds that RT should be a tourism activity that takes place in nonurban areas and takes agricultural production and rural life experience as tourism attractions.

From the system theory, it can be seen that RT can be developed because many driving factors form the result of joint promotion. The driving mechanism to promote the development of RT should be a controllable and open system, which is a multi-input, multioutput, and multicontrol variable set composed of many factors. This paper tries to combine the four systems of demand, supply, support, and intermediary, summarizes the driving forces of RT development in various regions in China according to the attribute characteristics of each factor, classifies them into internal driving forces and external driving forces, and constructs a driving mechanism model system according to this attribute (Figure 1). Each driving force factor jointly promotes the rapid development of RT according to different ways of action.

With the development of economy, people's material living standard and disposable income are gradually increasing, which makes people who have got rid of the problem of food and clothing pay attention to other aspects of life consumption, and the expenditure on basic necessities such as food accounts for less and less of the total expenditure. Postmodern tourism has gradually replaced mass tourism as a new way of tourism. And the postmodernity "perceptual experi- ence" can be realized through RT. Therefore, the group with postmodern consciousness has become the main source of RT. Media subsystem is the medium connecting RT destination and RT source. Although the media subsystem does not directly affect the development of RT, it influences the development of RT by influencing the demand power and the gravitational power. The essence of RT resources is "rural," while the essence of urban tourism resources is "modernity." According to the six elements of tourism: food, accommodation, transportation, travel, shopping, and entertainment, the products supplied by RT are divided into six types: sightseeing, transportation, diet, accommodation, shopping, and sports and entertainment.

The macroperspective of the local government, which promotes the development of RT by making policies, planning rural destinations, establishing effective management systems, and managing the RT industry, is referred to as the decision-making subsystem. The importance of scientific decision-making, a smooth management system, and a management system in ensuring the coordinated and steady development of RT cannot be overstated. The support subsystem primarily supports the smooth development of RT from both the hardware and soft environments, in order to increase the appeal of RT and RT products, and it serves as an important auxiliary role in enhancing the supply subsystem's appeal in the driving mechanism. Finally, the decision subsystem is a powerful external driving force for RT development, which primarily refers to the attitude of local government toward the development of local RT, which will have an impact on other subsystems in the RT development driving mechanism.

3.2. Operation of RT Development Driving Mechanism. The operation process of RT-driven mechanism is a process in which each adaptive subject in RT system exerts initiative or adaptability to promote the evolution and development of the system. There are two main mechanisms in this operation process, namely, the cooperative operation mechanism of each adaptive agent and the individual-led operation mechanism of each adaptive agent. Through the network connection, form a joint force to jointly promote the development of RT. In this driving mechanism structure, all adaptive subjects have no hierarchy, tend to cooperate, and participate in RT together, serving the broad interests of the long-term development of RT.

In the stage of participation, the local government has become the core and adaptive subject of tourism development, so the basic contradiction of local government activities has become the first driving force. At the stage of development and consolidation, the tourism industry plays an important role in tourism development, and the basic contradiction of tourism industry activities ranks first. When the internal contradiction of an adaptive subject is unified, this contradiction will lose its main driving role in the development of RT system. When the contradiction of one adaptive subject is unified, the contradiction of the next adaptive subject that is not unified will become the dominant driving force for the development of RT system $[4,5]$. Under different RT destinations and different levels of RT development, 


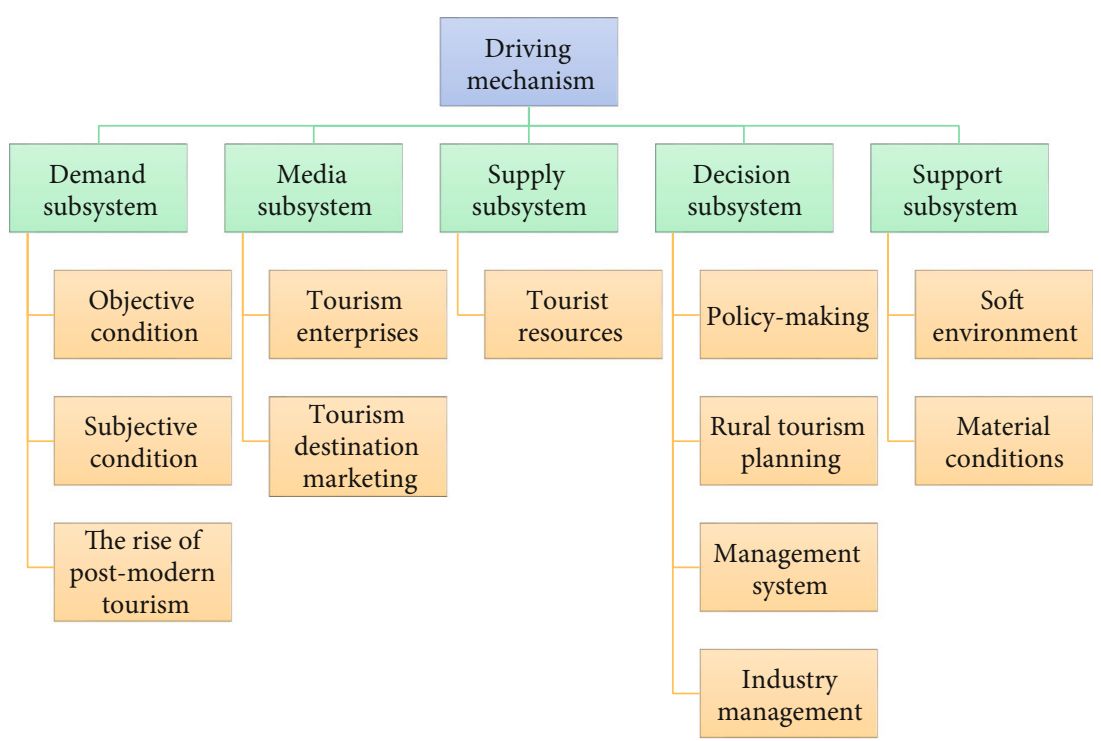

FIGURE 1: RT development driving mechanism model.

the relative power intensity and exposure degree of RT driving mechanism are also different.

The research on the driving mechanism of RT development is a complex systematic project. To reveal the action mechanism among the elements in the driving mechanism and the operating mechanism of the driving mechanism, it is far from enough to rely solely on qualitative research methods, but must resort to various research methods. On the basis of referring to relevant domestic research results and following the above-mentioned construction principles, this paper mainly starts from the source power that affects the development of RT, that is, from the perspective of data mining of demand subsystem and supply subsystem, selects certain evaluation factors, and describes and analyzes the running status, target realization degree, and overall benefits of RT in case areas.

PCA (principal component analysis) is an important multivariate statistical analysis method and the most commonly used feature extraction method, and this method is widely used in the fields of pattern recognition, image processing, comprehensive evaluation, fault diagnosis, and so on.

There are $p$ original indicators: $X_{1}, X_{2}, \cdots, X_{P}$, which are used to evaluate $n$ units, and there are $n_{p}$ data. PCA is used to combine these original indexes into a new independent comprehensive index: $Y_{1}, Y_{2}, \cdots, Y_{P}$, which is a linear function of the original indexes:

$$
\left\{\begin{array}{l}
Y_{1}=a_{11} X_{1}+a_{21} X_{2}+\cdots+a_{p 1} X_{p} \\
Y_{2}=a_{12} X_{1}+a_{22} X_{2}+\cdots+a_{p 2} X_{p} \\
\vdots \\
Y_{p}=a_{1 p} X_{1}+a_{2 p} X_{2}+\cdots+a_{p p} X_{p}
\end{array}\right.
$$

Through mathematical calculation, the total variance of $p$ original indexes can be decomposed into the sum $\lambda_{1}, \lambda_{2}$, $\cdots, \lambda_{P}$ of the variances of $p$ unrelated comprehensive indexes, and the variance of the first comprehensive index $Y_{1}$ reaches the maximum, the variance of the second comprehensive index $Y_{2}$ is large, and so on.

Transform the original coordinate system into a new orthogonal coordinate system. Among

$$
\begin{gathered}
Y_{1}=X_{1} \cos \theta+X_{2} \sin \theta \\
Y_{2}=-X_{1} \sin \theta+X_{2} \cos \theta, \\
{\left[\begin{array}{c}
Y_{1} \\
Y_{2}
\end{array}\right]=\left[\begin{array}{cc}
\cos \theta & \sin \theta \\
-\sin \theta & \cos \theta
\end{array}\right]\left[\begin{array}{l}
X_{1} \\
X_{2}
\end{array}\right] .}
\end{gathered}
$$

Through the rotation transformation, the dispersion degree of $n$ sample points in the $Y_{1}$ axis direction is the largest, that is, the variance of $Y_{1}$ is the largest, the variable $Y_{1}$ represents most information of the original data, $Y_{1}$ is called the first principal component, and $Y_{2}$ is called the second principal component.

The continuous improvement of the living standard of ordinary people reflects the need and necessity of travel. The desire to travel grows in tandem with the satisfaction of material life. As a result, when users want to visit a new city, it is critical to provide personalized tourist attractions tailored to their specific needs and preferences. The algorithm block diagram for personalized tourist attraction recommendations based on collective wisdom is shown in Figure 2.

To complete the personalized recommendation of scenic spots, the recommendation problem is regarded as a classification problem in order to find scenic spots that are similar to the user's interactive selection. The candidate recommended scenic spots pass the designed personalized scenic spot recommendation model when combined with scenic spots that have explicit user interaction. The user's location is used as context information to optimize the candidate list of recommended scenic spots because the user's location can affect the current user's scenic spot selection. Finally, the 


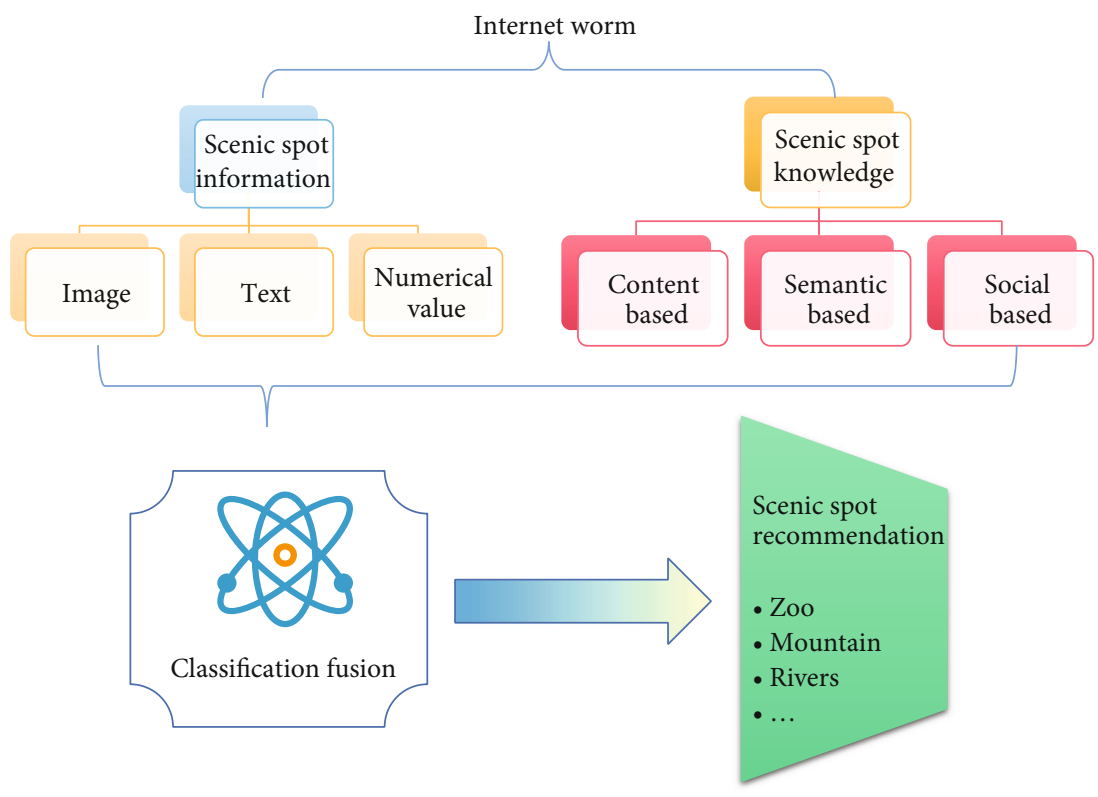

FIGURE 2: Block diagram of personalized recommendation of tourist attractions based on collective wisdom.

personalized scenic spot recommendation is displayed as a sorted list of scenic spots. Because scenic spots are represented by numerical values, social similarity is expressed by the distance between scores, i.e., the Euclidean distance is used to measure social-based knowledge similarity among scenic spots, which is as follows:

$$
D_{\text {Social }}=D_{\mathrm{Eu}}\left(R^{i}, R^{j}\right)=\exp \left(-\frac{\left\|R^{i}-R^{j}\right\|}{\sigma^{2}}\right),
$$

where $R^{i}$ represents the characteristics of scenic spot $A_{i}$ based on social knowledge and $\sigma$ represents the distance constant, which is set to 0.2 .

Because users need a more intuitive result, the expression of ordered list of scenic spots is adopted here, and the output of the previous hard classification result is converted into probability, and the classification function $f$ can be changed into probability, and then, the probability of each scenic spot $A_{i}$ is expressed as follows:

$$
P^{A}\left(A_{i}\right) \approx \frac{1}{1+\exp \left(-f\left(A_{i}\right)\right)} .
$$

The ranking of recommended scenic spots is considered to be related to the probability of content-based and situational context-based. Finally, the ranking score of scenic spots $A_{i}$ is calculated as follows:

$$
S(A)=\alpha_{S} P^{A}\left(A_{i}\right)+\left(1-\alpha_{S}\right) P^{G}\left(A^{i}\right) .
$$

Among them, $0<\alpha_{S}<1$ is set to 0.8 according to the experience value $\alpha_{S}$ to balance the influence of content and context. Therefore, the personalized scenic spot recommendation based on collective wisdom fusion not only considers the user's preferences but also combines the user's situational positioning information.

\section{System Simulation Results and Analysis}

The collection, extraction, and follow-up analysis of RT data are all influenced by the quality of segmentation results. The monthly index (monthly index = monthly release volume of Weibo/average monthly release volume of Weibo in that year) [6] is used as the benchmark coordinate in this paper, and the release volume of Weibo in $\mathrm{H}$ city including the keywords of farmhouse RT is counted (Figure 3).

Figure 3 shows that the seasonal distribution pattern of RT Weibo release volume is characterized by "three peaks," with a high center and low ends. Although there will be a short-term lag in the release of the farmhouse RT for fellow travelers on Weibo, the distribution of the release volume on Weibo can be used to divide the peak season and off-season of the farmhouse RT for tourists. The farmhouse RT is busiest in the months of April and May, as well as August and October. Weibo's captured time period distribution information is depicted in Figure 4.

From Figure 4, it can be clearly seen that the release volume of Weibo in the weekend of one week is obviously higher than that of Weibo in the working days from Monday to Friday, and it is obviously on the rise from Friday to Sunday, reaching the maximum of 2053 on Sunday. Affected by working days, the number of farmhouse tourism dropped sharply compared with weekends. From Monday to Tuesday, the number published in Weibo began to decline to a certain extent, reaching the minimum of 1183 on Tuesday, while from Wednesday to Thursday, it kept a small fluctuation trend.

The purpose of personalized scenic spot similarity model is to recommend scenic spots similar to users' explicit feedback. This experiment is aimed at testing the accuracy of 


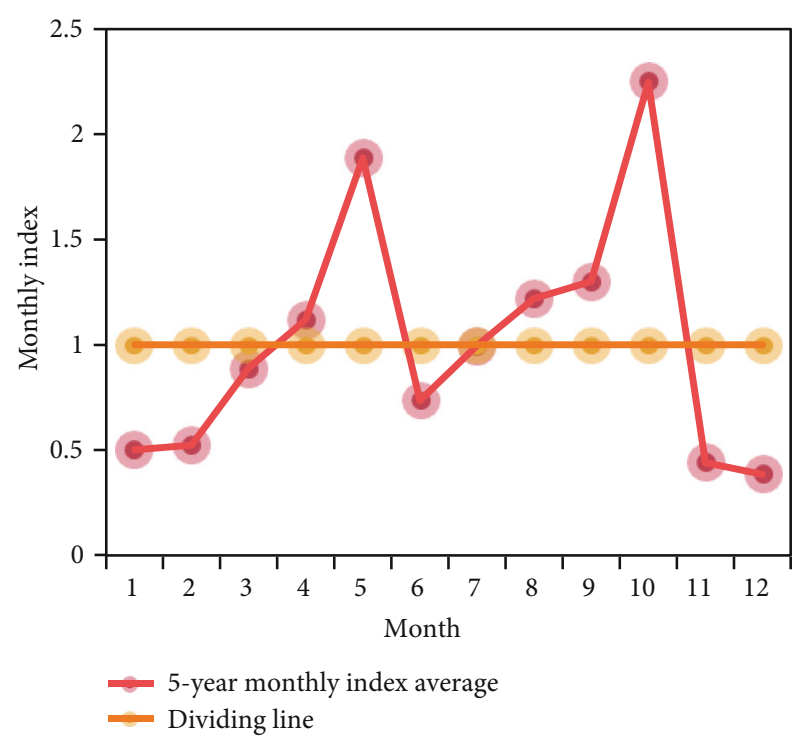

FIGURE 3: Average distribution of monthly index of related Weibo releases.

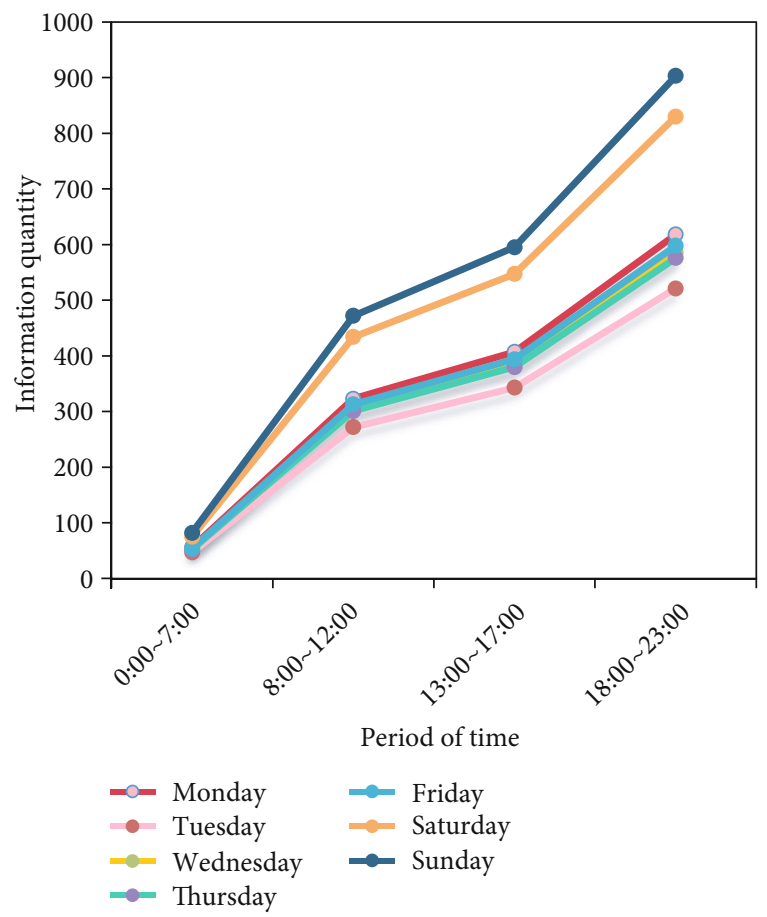

Figure 4: Distribution of Weibo release time period within one week.

personalized scenic spot similarity model in recommending scenic spots. In the constructed database, a group of pseudocorrelation samples are selected as marking data to verify the performance of the recommendation model. Figure 5 shows the accuracy of the proposed model and comparison method for different query terms. Among them, PL is preference level; MRVI is manifold regularization based on visual information; MRTI is manifold regularization based on text information; PS_L is personalized scenic spot recommenda-

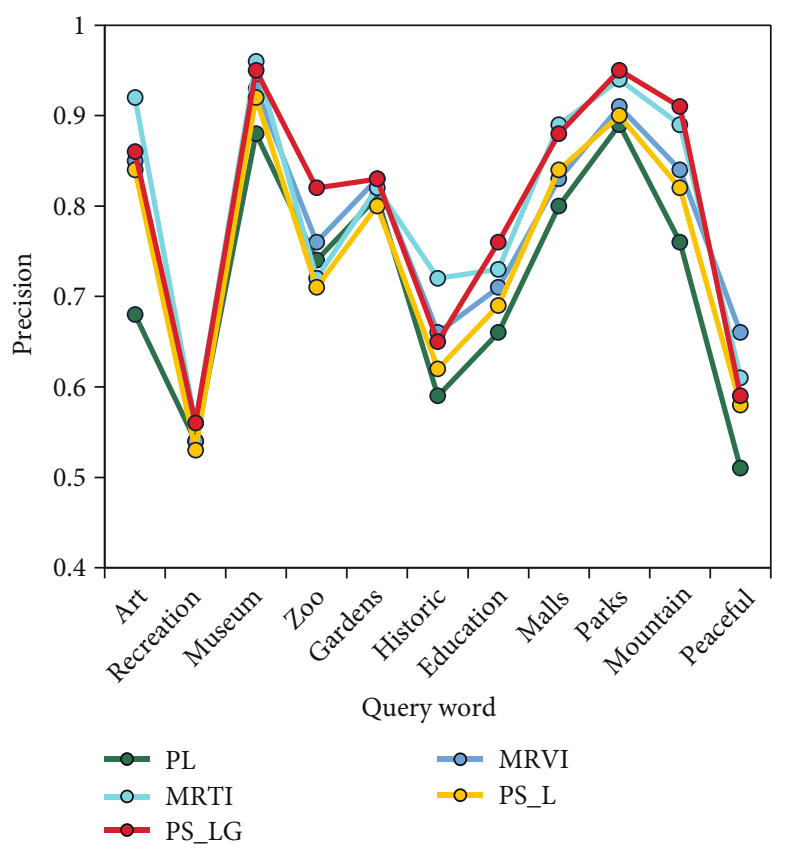

FIgURE 5: The accuracy of the proposed model and comparison method for different query terms.

tion model without weight learning; PS_LG is personalized scenic spot recommendation model with weight learning.

The experimental results show that after searching and sorting based on query words, sorting according to preference level can only show users the scenic spots with the highest scores, while ignoring the use of content-based and semantic-based knowledge to realize the personalization of scenic spot recommendation. Since adaptive weight learning can find more similar scenic spots by effectively highlighting an important feature in the process of knowledge fusion, the model with weight learning shows the best accuracy.

Figure 6 gives a summary of the real and reliable user travel data obtained in different cities.

The similarity model takes into account the recommendation of scenic spots based on content and semantic knowledge, and data scarcity has no bearing on this. Furthermore, it incorporates preference scoring, i.e., multimodal heterogeneous knowledge. As a result, the personalized similarity model outperforms the other two methods of comparison. Furthermore, the personalized similarity model shows that when the marked positive sample data is 2 , the accuracy rate is the highest. This paper tests the feasibility and effectiveness of the established system data mining model before data calculation and system simulation to ensure that it corresponds to the actual situation of RT development in $\mathrm{H}$ city. The simulation results are compared to the real data of RT development in $\mathrm{H}$ city in 2019 and 2020 to verify the model's effectiveness. Figures 7 and 8 depict the model test results. RTI stands for rural tourism income (10,000 yuan); TA V stands for total agricultural output value $(10,000$ yuan); AR A stands for area of rural tourist attractions (square kilometers); NRT stands for number of rural tourists (10,000 yuan); and PN F stands for per capita net income of farmers (ten thousand yuan). 


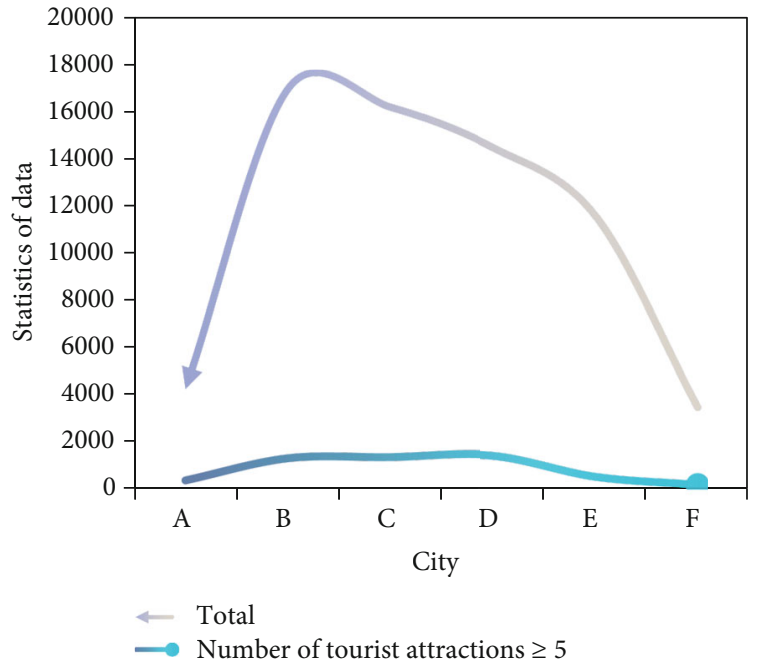

FIgURE 6: Statistics of real and reliable user travel data obtained in different cities.

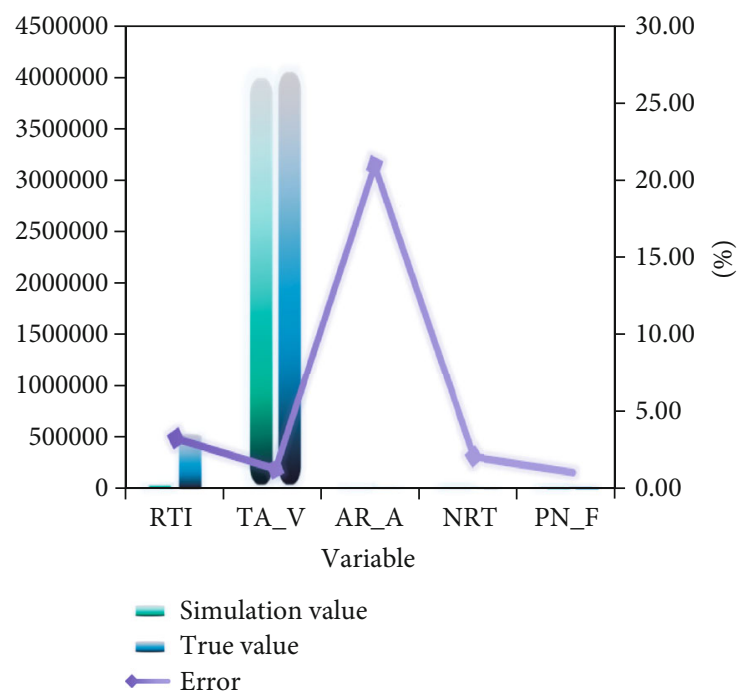

FIGURE 7: Test results of data mining model of driving mechanism system (2019).

By comparing the simulation results with the actual data, it is found that the average error between the simulation values and the historical values is between $-3.22 \%$ and $9.37 \%$. The results show that the model has a high degree of fitting, which can truly and effectively reflect the operation of RT system in $\mathrm{H}$ city and can be used for the simulation and prediction of RT development in $\mathrm{H}$ city.

This paper divides the driving mechanism modes of RT into five types and compares and analyzes the simulation results of each mode by adjusting the related variables in the simulation stage in order to investigate the development and changes of RT in $\mathrm{H}$ city under different policies. The control variables of the industrial economic subsystem in this paper are tourism investment rate and agricultural investment rate. As the social and cultural subsystem's regulatory variables, choose the number of tourism service

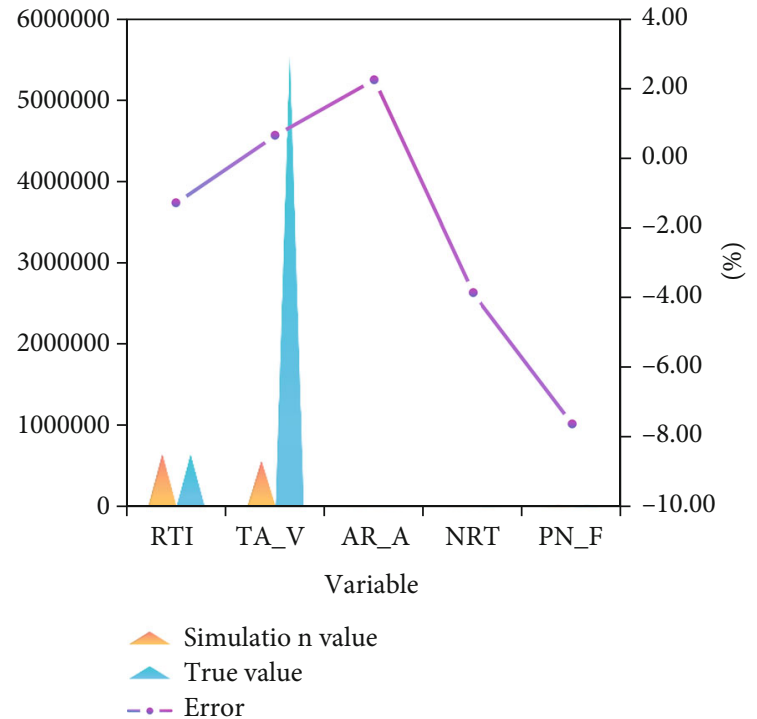

FIGURE 8: Test results of data mining model of driving mechanism system (2020).

employees and the proportion of cultural and educational expenditures; as the resource and environment subsystem's control variables, choose the proportion of environmental protection expenditure and tourism publicity investment. Figure 9 depicts the parameter changes of control variables in various driving modes.

I_M is industry-led driving mode; $\mathrm{C} \_\mathrm{M}$ is culture-led driving mode; E_M is environment-led driving mode; ISR $\mathrm{M}$ is industrial economy-social culture-resource environment coordinated development model.

The simulated fruit is basically in line with the actual data before 2019, and the development trend is generally consistent. RT in $\mathrm{H}$ city shows a steady growth. From the simulation diagram, it can be seen that RT revenue shows a steady growth trend under the natural development mode, but the growth rate slows down. The total output value of agriculture increased greatly with the development of RT.

In view of the difference of growth range of different subsystems, to realize the coordinated development of RT in $\mathrm{H}$ city, different development strategies should be put forward for different subsystems within the system, and no single adjustment can be made. While paying attention to the economic benefits of RT, it is also necessary to pay attention to environmental protection of resources and ensure that the number of tourists is adapted to the carrying capacity of the environment. Only by increasing efforts in promoting farmers' employment and improving farmers' cultural level can RT achieve sustainable development.

There are fresh air, clean water sources, and pleasant climate in rural areas that cities cannot match, and there are also a large number of idle land, idle homestead, and seasonal surplus labor force. RT should apply the concept of sharing economy, innovate tourism experience sharing economy practices such as sharing homes, sharing farms, and sharing farmhouses, make use of the intervention of Internet, Internet of Things, and other scientific and technological means, revitalize rural idle resources, and promote 


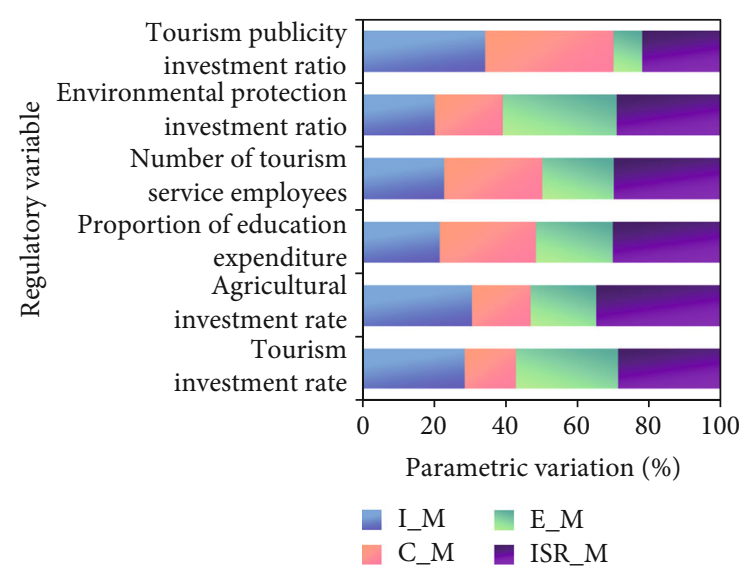

Figure 9: Changes of control parameters in the model.

the realization and appreciation of rural resources in the context of the expanding antiurbanization market.

Media subsystem is a bridge connecting RT destination and tourist destination, through which both sides can directly exchange information on tourism supply and demand. Therefore, the governments or enterprises of RT destinations in various districts (counties) of $\mathrm{H}$ city can cooperate with other tourism enterprises, break administrative boundaries, establish information interaction platforms, exchange tourists among tourism enterprises, establish joint preferential measures, set up green channels, etc., which not only saves a lot of marketing costs but also is conducive to the common cultivation of tourist markets.

\section{Conclusion}

Through the above research and analysis, the driving forces of core scenic spots, endogenous resources, urban driving forces, and traffic driving forces are now the main driving forces of RT development, and external and internal driving forces jointly promote the development of RT. Using data mining, the RT driving mechanism system is divided into three subsystems, namely, industrial economy subsystem, resource environment subsystem, and social culture subsystem, and the conceptual model of RT development driving mechanism is constructed. Actual data from RT development in $\mathrm{H}$ city is used to test the model. The results show that the predicted and actual values are nearly identical, indicating that the model can be used to simulate RT development through the test. The driving forces behind the development of RT are the demand and supply subsystems. They interact and constrain the other three subsystems, forming a joint force to jointly promote the operation of the RT driving mechanism, which is critical to the longterm survival of RT.

\section{Data Availability}

The data used to support the findings of this study are included within the article.

\section{Conflicts of Interest}

The author does not have any possible conflicts of interest.

\section{References}

[1] F. Zhou, W. Zhang, W. Su, H. Peng, and S. Zhou, "Spatial differentiation and driving mechanism of rural water security in typical "engineering water depletion" of karst mountainous area-a lesson of Guizhou, China," Science of the Total Environment, vol. 793, no. 3, article 148387, 2021.

[2] X. Gao-Zeller, X. Li, F. Yang, and W. Zhu, "Driving mechanism of CSR strategy in Chinese construction companies based on neo-institutional theory," KSCE Journal of Civil Engineering, vol. 23, no. 5, pp. 1939-1951, 2019.

[3] Y. Shao, Q. Jiang, C. Wang, M. Wang, L. Xiao, and Y. Qi, "Analysis of critical land degradation and development processes and their driving mechanism in the Heihe River Basin," The Science of the Total Environment, vol. 716, article 137082, 2020.

[4] A. Taufiq, A. J. Effendi, I. Iskandar, T. Hosono, and L. M. Hutasoit, "Controlling factors and driving mechanisms of nitrate contamination in groundwater system of Bandung Basin, Indonesia, deduced by combined use of stable isotope ratios, CFC age dating, and socioeconomic parameters," Water Research, vol. 148, pp. 292-305, 2019.

[5] P. O.-W. Adjei, D. Buor, and P. Addrah, "Ecological health effects of rural livelihood and poverty reduction strategies in the Lake Bosomtwe basin of Ghana," Geo Journal, vol. 82, no. 3, pp. 609-625, 2017.

[6] R. Wang, M. Dai, Y. Ou, and X. Ma, "Residents' happiness of life in rural tourism development," Journal of Destination Marketing and Management, vol. 20, no. 1, article 100612, 2021.

[7] C. Liu, X. Dou, J. Li, and L. A. Cai, “Analyzing government role in rural tourism development: an empirical investigation from China," Journal of Rural Studies, vol. 79, pp. 177-188, 2020.

[8] Y. Kortoci and M. Kortoci, "The assessment of the rural tourism development in the Valbona Valley National Park," Tourism Economics, vol. 23, no. 8, pp. 1662-1672, 2017.

[9] S. M. Rasoolimanesh, J. L. Roldán, M. Jaafar, and T. Ramayah, "Factors influencing residents' perceptions toward tourism development: differences across rural and urban world heritage sites," Journal of travel research: The International Association of Travel Research and Marketing Professionals, vol. 56, no. 6, pp. 760-775, 2017.

[10] A. Scaglione and D. Mendola, "Measuring the perceived value of rural tourism: a field survey in the western Sicilian agritourism sector," Quality and Quantity, vol. 51, no. 2, pp. 745-763, 2017.

[11] S. Snyman, "African tourism industry employees: expenditure patterns and comparisons with other community members," Journal of Sustainable Tourism, vol. 27, no. 6, pp. 788-804, 2019.

[12] M. Strzelecka, B. B. Boley, and C. Strzelecka, "Empowerment and resident support for tourism in rural Central and Eastern Europe (CEE): the case of Pomerania, Poland," Journal of Sustainable Tourism, vol. 25, no. 4, pp. 554-572, 2017.

[13] S. M. Rasoolimanesh, C. M. Ringle, M. Jaafar, and T. Ramayah, "Urban vs. rural destinations: residents' perceptions, community participation and support for tourism development," Tourism Management, vol. 60, pp. 147-158, 2017. 
[14] L. Zhou, E. Chan, and H. Song, "Social capital and entrepreneurial mobility in early-stage tourism development: a case from rural China," Tourism Management, vol. 63, pp. 338350, 2017.

[15] I. Dinis, O. Simões, C. Cruz, and A. Teodoro, "Understanding the impact of intentions in the adoption of local development practices by rural tourism hosts in Portugal," Journal of Rural Studies, vol. 72, pp. 92-103, 2019.

[16] S. R. M. Reid, "University extension and rural tourism enterprise development: a rare Australian case," The Journal of Hospitality Leisure Sport and Tourism, vol. 23, pp. 10-17, 2018.

[17] P. Longart, E. Wickens, W. Ocaña, and V. Llugsha, "A stakeholder analysis of a service learning project for tourism development in an Ecuadorian rural community," The Journal of Hospitality Leisure Sport and Tourism, vol. 20, pp. 87-100, 2017.

[18] Y. Wang, "Institutional interaction and decision making in China's rural development," Journal of Rural Studies, vol. 76, pp. 111-119, 2020.

[19] T. Li, Q. Li, and J. Liu, "The spatial mobility of rural tourism workforce: a case study from the micro analytical perspective," Habitat International, vol. 110, no. 7, article 102322, 2021.

[20] C. Qian, N. Sasaki, D. Jourdain, S. M. Kim, and P. G. Shivakoti, "Local livelihood under different governances of tourism development in China - a case study of Huangshan mountain area," Tourism Management, vol. 61, pp. 221-233, 2017.

[21] F. Randelli and F. Martellozzo, "Is rural tourism-induced builtup growth a threat for the sustainability of rural areas? The case study of Tuscany," Land Use Policy, vol. 86, no. C, pp. 387-398, 2019.

[22] Z. Mottiar, K. Boluk, and C. Kline, "The roles of social entrepreneurs in rural destination development," Annals of Tourism Research, vol. 68, pp. 77-88, 2018.

[23] Y. He, X. Gao, R. Wu, Y. Wang, and B. R. Choi, "How does sustainable rural tourism cause rural community development?," Sustainability, vol. 13, no. 24, p. 13516, 2021.

[24] T. Huang, X. I. Jian-Chao, and G. E. Quan-Sheng, "Livelihood differentiation between two villages in Yesanpo Tourism District in China," Journal of Mountain Science, vol. 14, no. 11, pp. 2359-2372, 2017.

[25] L. Yanjuan, "Cognitive diagnosis of cultural and rural tourism convergence," Translational Neuroscience, vol. 10, no. 1, pp. 19-24, 2019.

[26] O. M. Ghoochani, R. Torabi, M. Hojjati, M. Ghanian, and M. Kitterlin, "Factors influencing Iranian consumers' attitudes toward fast-food consumption," British Food Journal, vol. 120, no. 2, pp. 409-423, 2018. 